\title{
THE INTERPRETATION OF XUV SOLAR RADIATION
}

\author{
LEO GOLDBERG \\ Harvard College Observatory, Cambridge, Massachusetts 02138, U.S.A.
}

\section{Introduction}

Solar ultraviolet and X-radiation arriving at the Earth is emitted mainly by highlycharged ions in the outer layers of the Sun, where departures from thermodynamic equilibrium are extreme. Hence the diagnosis of physical conditions from spectral intensities entails the use of accurate cross sections for a variety of physical processes governing the excitation and ionization of emitting ions. Now that the full potential of satellites for solar data acquisition has begun to be realized, the need for atomic data of all kinds is more acute than ever before. In this review, I shall illustrate the importance of laboratory and theoretical investigations for the analysis of solar ultraviolet and X-ray data by referring to five problems to which space experimenters are currently devoting a great deal of attention:

(1) the temperature minimum at the interface between the photosphere and the chromosphere,

(2) the temperature and density profiles of the low chromosphere,

(3) the transition zone at the chromosphere-corona interface,

(4) the corona,

(5) solar flares.

\section{The Temperature Minimum}

Visible solar radiation is emitted from layers at an average depth of a few hundred kilometers below the level of the temperature minimum. As the continuous opacity increases towards shorter wavelengths, ultraviolet continuum radiation originates from progressively higher levels in the atmosphere and emerges from the region of the temperature minimum approximately in the wavelength range $2000-1400 \AA$. The temperature distribution in the atmosphere may be inferred from absolute measurements of spectral intensities in this region provided that the sources of opacity and their cross sections are known (Gingerich, 1970). The principal absorbers are $\mathrm{Al}, \mathrm{Si}$, $\mathrm{C}$ and $\mathrm{S}$, and a variety of theoretical and experimental cross sections are used to compute their photoionization rates. Below $1700 \AA$, the observed intensity distribution can be matched with that calculated for a model atmosphere in local thermodynamic equilibrium (LTE). Beyond $1700 \AA$, the calculated intensities are as much as an order of magnitude greater than the observed intensities. This discrepancy was at first thought to require additional sources of opacity; among those conjectured and found wanting were quasi-molecular absorption by $\mathrm{H}_{2}$ and wing absorption by Lyman- $\alpha$. Heavy blanketing by atomic absorption lines and $\mathrm{CO}$ bands and boundfree absorption by iron may account for the discrepancy. 
It is by no means clear that the assumption of LTE is fully justified for the temperature minimum. Recent calculations by Cuny (1970) show in fact that whereas LTE is a valid approximation for the calculation of the populations of the metastable levels of $\mathrm{Si}, \mathrm{S}$, and $\mathrm{C}$, the degree of ionization of these elements shows marked departures from LTE and must be calculated with the aid of appropriate cross sections for photoionization and radiative recombination.

\section{The Low Chromosphere}

The Lyman continuum is formed at heights in the solar atmosphere intermediate between those of the temperature minimum and the transition zone chromospherecorona. The first spatially-resolved observations of the Lyman continuum, which were made with the Harvard College Observatory's spectroheliometer flown aboard OSO-IV, have been used to derive a model of the upper chromosphere. The observations show that whereas the color temperature of the Lyman continuum at the disk center is $8300 \mathrm{~K}$, the brightness temperature at the series limit is $6500 \mathrm{~K}$. Furthermore, the continuum progresses smoothly from limb darkening at $911 \AA$ to limb brightening at $670 \AA$, the cross-over occurring at approximately $740 \AA$. Noyes and Kalkofen (1970) have succeeded in accounting for the observations with a homogeneous model of the upper chromosphere derived with the aid of radiative transfer calculations for a plane parallel atmosphere in hydrostatic equilibrium. In the construction of the model, the transfer equations for the Lyman continuum and the $\mathrm{H} \alpha$ line are made consistent with the equations of statistical equilibrium for a model hydrogen atom with three discrete levels and a continuum. A variety of experimental and theoretical cross-sections for radiative and collisional transitions are available for these calculations.

The calculated variation of the temperature, pressure, and number densities is shown in Figure 1. The temperature range in which the model is determined by the Lyman continuum data is $9400-7400 \mathrm{~K}$. It is found that at $\tau(912 \AA)=1$, the electron temperature is $8300 \mathrm{~K}$ and the ionization temperature $6500 \mathrm{~K}$. Thus the departure coefficient $b_{1}$ at the same depth is 200 . Both $T_{e}$ and $b_{1}$ increase upward, but the rates of increase are different. Hence the source function $B\left(T_{e}\right) / b_{1}$ decreases upward at $912 \AA$ and increases upward at $667 \AA$. The model from $T_{e}=7400$ to $T_{e}=5800$ fits brightness measurements at millimeter wavelengths (Linsky and Avrett, 1970) and eclipse measurements of electron densities (Henze, 1969), and at still lower levels the model has been joined to that of Gingerich et al. (1971).

Because of higher electron density, conditions in active regions would be expected to approximate more closely those in thermodynamic equilibrium. New data on the Lyman continuum from OSO-VI (Noyes, 1970) show indeed that the brightness temperature at the series limit is several hundred degrees higher in active regions than in quiet regions and furthermore that the electron temperature is lower by about $500 \mathrm{deg}$. Consequently, the departure coefficient $b_{1}$ is smaller by an order of magnitude. 


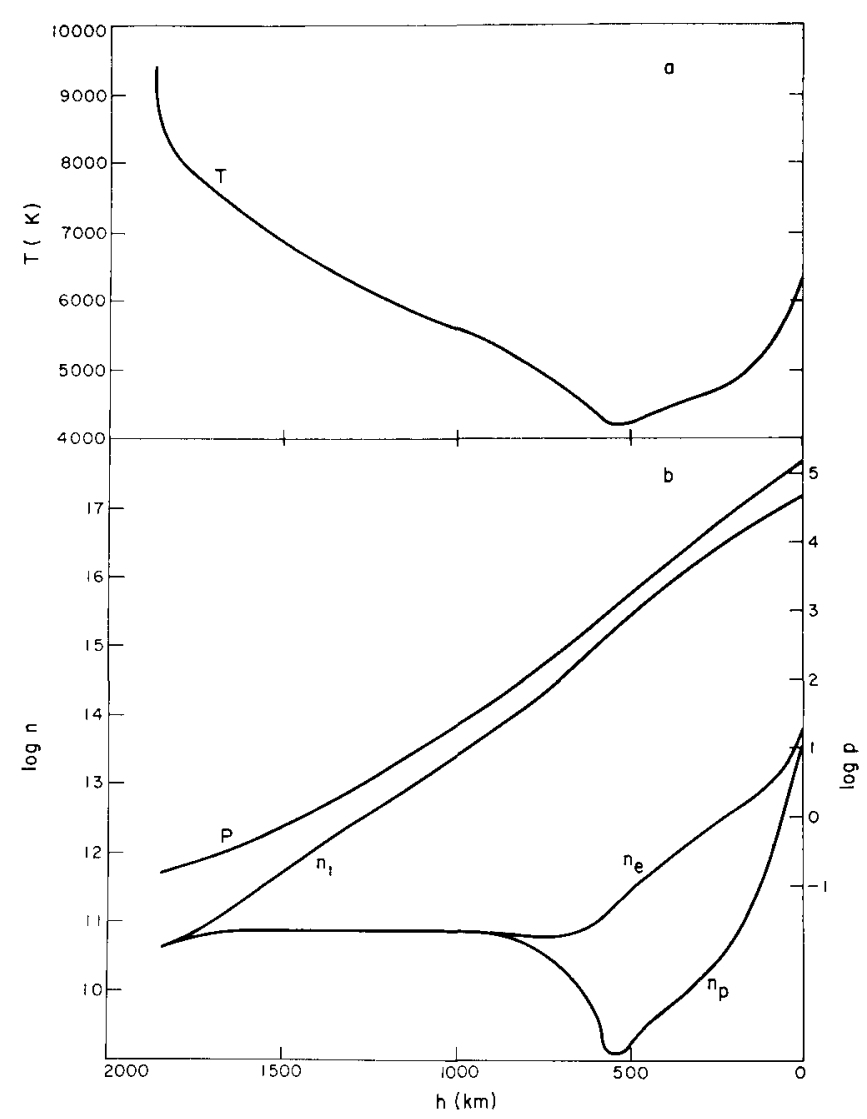

Fig. 1. (a) The temperature $T$ as a function of the height $h$ from the upper photosphere to the beginning of the transition zone chromosphere-corona. (b) The total pressure $P$, and the number densities of ground-state $\mathbf{H}$ atoms $n_{1}$, of electrons $n_{e}$, and of protons $n_{p}$ (Noyes and Kalkofen, 1970).

\section{The Transition Zone Chromosphere-Corona}

One of the major contributions of space research to solar physics has been the derivation, with some degree of certainty, of the shape and location of the transition zone chromosphere-corona. The transition zone is so thin and the temperature gradient within it is so steep that only the resonance lines, which radiate in the far ultraviolet, are strong enough to be observed. Subordinate lines which might otherwise be seen in the visible spectrum are too weak to be detected. One method of deriving the structure of the transition zone, which has been used by several investigators, makes use of measurements of the total flux from the whole Sun in the resonance lines of abundant elements in several stages of ionization, particularly $\mathrm{H}, \mathrm{He}, \mathrm{C}, \mathrm{N}, \mathrm{O}, \mathrm{Ne}$, $\mathrm{Mg}, \mathrm{Si}$ and $\mathrm{S}$. It is usually assumed in the analysis that the resonance lines are optically thin and that the upper levels of the transitions are excited by electron impact from the ground level and de-excited by spontaneous emission. The degree of ioni- 
zation is calculated by equating the rate of collisional ionization by electron impact with that of radiative and dielectronic recombination. The relevant atomic parameters, which are believed to be known within a factor of two, are the rate coefficients for excitation, ionization and recombination and the oscillator strengths.

Models of the transition zone derived from whole-Sun fluxes have been used by Withbroe (1970a) to predict intensity profiles for resonance lines on both sides of the solar limb and compared with spatially-resolved intensities from the Harvard experiment on OSO-IV. Only relatively minor changes in the previously derived models are necessary to give a good account of the center-limb behavior of lithiumlike resonance lines formed in the quiet equatorial zone. The same model also reproduces the intensities of resonance lines of beryllium-like ions formed in the lower half of the transition zone, except that when the wavelengths are below $912 \AA$, their limb intensities appear to be attenuated by spicules, which are optically thick in the Lyman continuum (Withbroe, 1970b).

Figure 2 shows the model adopted by Withbroe and joined to the high-temperature end of the chromospheric model in Figure 1. A remarkable feature of the model, which was first pointed out by Athay (1966), is that the temperature gradient in the

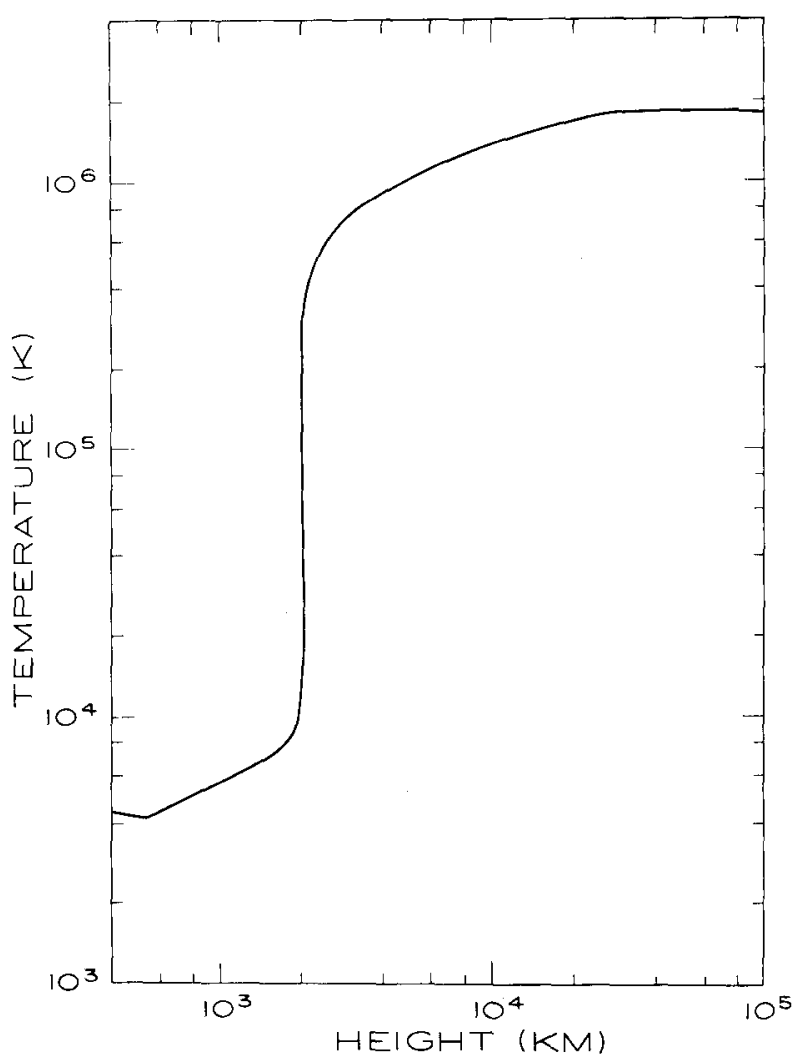

Fig. 2. The temperature as a function of height in the chromosphere and corona. 
range of temperatures between $10^{5}$ and $10^{6} \mathrm{deg}$ is given very closely by the equation of conductive equilibrium, viz.,

$$
(\mathrm{d} T / \mathrm{d} h)^{-1}=C \cdot T^{5 / 2} .
$$

The conductive flux model for the transition zone is joined to an isothermal corona whose temperature $T_{C}=2 \times 10^{6} \mathrm{~K}$ is found from the intensity ratio above the limb of two lines with different ionization potentials, e.g., SixII, $\lambda 499$ and $\operatorname{Mgx} \lambda 625$. The parameter $C$ is found to be $10^{-12.0}$ which corresponds to a conductive flux $F_{C}=$ $=6 \times 10^{5} \mathrm{erg} / \mathrm{cm}^{2} / \mathrm{sec}$. In addition to $T_{C}$ and $C$, the third parameter defining the model is the product $P_{0}=N_{e} T_{e}=7 \times 10^{14}$.

The temperature structure of the transition zone may also be inferred from the relative intensities of emission lines observed in rocket spectra just above the limb and at the center of the disk (Burton et al., 1970). Since each line is formed over a narrow range of heights, the intensity ratios depend only upon the heights of the emitting regions of different ions and are independent of atomic data and absolute intensity calibration. Within the accuracy of the data, the structure found from optically thin lines agrees with that derived by the other two methods. But optically thick lines seem to be radiated several thousand kilometers higher on the average and their emission is spread over a much greater range in height, which suggests that the optically thick lines are radiated by spicules.

The OSO observations of far ultraviolet emission lines (Noyes et al., 1970) show that there are significant variations in the structure of the transition zone from one region to another. On the average, the pressure in an active region is increased by about a factor of 5 over the quiet Sun value, the temperature gradient is increased by a like amount and the temperature of the corona is also increased to about $2.5 \times 10^{6} \mathrm{~K}$. Hence the conductive flux and therefore the mechanical heat input over active regions are also increased on the average by a factor of 5 . The new data from OSO-VI, consisting of spectra at different points in the active region, also show large point-topoint variations in both the conductive flux (or temperature gradient) and the coronal temperature (Noyes, 1970).

\section{The Corona}

In this section I shall sketch some of the recently developed diagnostic techniques for the determination of coronal temperatures and electron densities and give some results of their application to ultraviolet and X-ray data.

\section{A. CORONAL TEMPERATURES}

Temperature models of the chromosphere-corona derived both from whole-Sun fluxes and spatially-resolved intensities suggest that the quiet corona is relatively isothermal within rather narrow limits, at leasi in the inner regions out to about $R=1.3 R_{\odot}$. Assuming that the corona is in fact isothermal, Withbroe (1971) has shown that the temperature may be determined from ratios of intensities above the limb of resonance 
lines of two different elements with known relative abundance. The intensity of an optically thin ultraviolet emission line is given by

$$
I(\varrho)=1.73 \times 10^{-16} \mathrm{Agf} \int_{0}^{\infty} G\left(T_{e}\right) n_{e}^{2} \mathrm{~d} h / \mu,
$$

where $\varrho$ is the distance from the center of the Sun in units of the solar radius $R_{\odot}, A$ is the abundance of the line-forming element relative to hydrogen, $g$ is the mean Gaunt factor for collisional excitation, $f$ is the oscillator strength of the line, $G\left(T_{e}\right)$ is the temperature dependent part of the function that gives the fractional concentration of the element in a particular state of excitation and ionization, $n_{e}$ is the electron density, $r$ is the distance from the center of the Sun and $\mu=\left[r^{2}-\left(\varrho R_{\odot}\right)^{2}\right]^{1 / 2} / 2 r$.

If the corona is assumed to be isothermal in the plane defined by the line of sight and the center of the Sun, the ratio of the intensities of two different spectral lines, e.g., $\lambda 499 \mathrm{SixII}$ and $\lambda 625 \mathrm{MgX}$ is given by

$$
\frac{I(\varrho)_{\mathrm{Si} \mathrm{XII}}}{I(\varrho)_{\mathrm{MgX}}}=\frac{A_{\mathrm{Si}}}{A_{\mathrm{Mg}}} \frac{\left[f g G\left(T_{C}\right)\right]_{\mathrm{Si} \mathrm{XII}}}{\left[f g G\left(T_{C}\right)\right]_{\mathrm{Mg} \mathrm{X}}}=\frac{A_{\mathrm{Si}}}{A_{\mathrm{Mg}}} f\left(T_{C}\right) .
$$

If the relative abundances of the two elements and the atomic parameters are known, the ratio may be calculated as a function of the temperature alone and the temperature inferred from the observed ratio.

Figure 3 shows temperatures derived by Withbroe (1970c) from intensity ratios measured on spectroheliograms obtained from the Harvard OSO-IV experiment at different points around the limb at a constant height of 2 arc min. Each intensity ratio represents an average of 5-8 spectroheliograms recorded in a single orbit within about $1 \mathrm{hr}$. The relative abundances used in the temperature determinations were $\mathrm{Si}: \mathrm{Mg}: \mathrm{Ne}: \mathrm{O}=1.0: 0.8: 0.8: 11.2$. Considering that the observations encompass both active and quiet regions in which the Sixı intensity varied by a factor of 10 or more, the relatively small range in temperature, from $1.5-2.0 \times 10^{6} \mathrm{~K}$, is noteworthy. The average value is about $1.8 \times 10^{6} \mathrm{~K}$, in good agreement with earlier determinations based on ultraviolet fluxes from the whole Sun. The spatial resolution of the OSO-IV data is 1 arc min and the amplitude of the temperature variation is almost certain to be increased when data of higher spatial resolution become available.

\section{B. ELECTRON DENSITIES}

Withbroe (1970c) derives electron densities from the same OSO-IV data by assuming that the variation of $n_{e}$ along any radius vector in the plane defined by the line of sight and the center of the Sun is given by the hydrostatic equilibrium formula with constant temperature $T_{C}$. Using a modified form of Equation (2) with observed absolute line intensities, and values of $T_{C}$ derived by the method of the previous section, he calculates the quantity $A\left(\left\langle n_{e}^{2}\right\rangle\right)^{1 / 2}$ for several lines as a function of position angle at a constant height of 2 arc min above the limb. $K$-coronameter data obtained on the same day with about the same spatial resolution at the High Altitude Observatory 


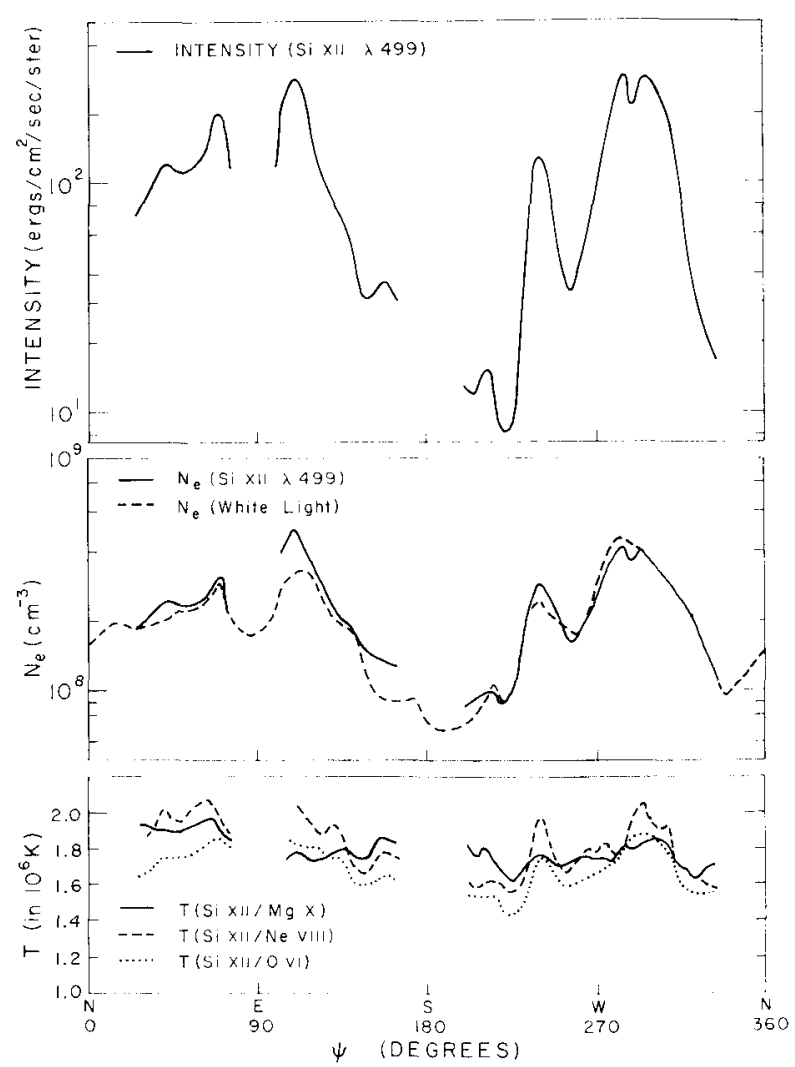

Fig. 3. top: Intensity of $\lambda 499$ SixII two minutes of arc above the limb from OSO-IV data. middle: rms values of $N_{e}$ derived from intensity of $\lambda 499 \mathrm{SixII}$ (solid line) and average values of $N_{e}$ derived from K-coronameter data (dashed line), bottom: Temperatures derived from ratios of line intensities according to Equation (3) (Withbroe, 1970c).

station in Hawaii may also be used to derive values of $\left\langle n_{e}\right\rangle$. Figure 3 compares $\left\langle n_{e}\right\rangle$ derived from $K$-coronameter data with $\left(\left\langle n_{e}^{2}\right\rangle\right)^{1 / 2}$ from Si xII data with $\log A_{\mathrm{Si}}=-4.5$. In view of possible misalignments in orienting the UV and white light images, changes in coronal structure that may have occurred in the time between the different observations, the statistical noise in the UV data, and the simplifying assumptions used in the interpretation, the rms and average electron densities are in remarkable agreement over the range from $5 \times 10^{7} \mathrm{~cm}^{-3}$ to $4 \times 10^{8} \mathrm{~cm}^{-3}$. The intensity of the SixII line $\lambda 499$ varies around the limb by a factor of about 30 . Most of this variation appears to be caused by fluctuations in density rather than in iemperature, the intensity being roughly proportional to the square of the electron density.

According to Equation (2), the off-limb spectra may also be used to derive relative abundances if the temperature is known. It is best to determine $T_{C}$ from the $\mathrm{Si} / \mathrm{Mg}$ ratio because (a) the relative abundances of these elements are well determined and (b) the temperature is relatively independent of the abundance. Next the intensity 
ratios $\mathrm{Mgx} / \mathrm{O}$ vi, $\mathrm{Mgx} / \mathrm{NevH}$, and $\mathrm{NeviI} / \mathrm{O}$ vi may be used to derive relative abundances because the predicted ratios are essentially independent of $T_{C}$ from 1.2-3 million deg. Finally, by assuming an azimuthally symmetric corona and using $K$ coronameter observations to derive $\left\langle n_{e}\right\rangle$ Withbroe obtains the abundances of these elements relative to hydrogen. The results found are in very close agreement with those of other investigators both for the corona and the photosphere.

\section{ELECTRON DENSITIES From X-RAY SPECTRA}

Gabriel and Jordan (1969) have identified a series of strong lines in the HeI isoelectronic sequence with the forbidden magnetic dipole transition $2{ }^{3} \mathrm{~S}-1{ }^{1} S$. This line and the transitions $2{ }^{3} P_{1}-1{ }^{1} S$ and $2{ }^{1} P-1{ }^{1} S$ form a triplet which has been identified for the helium-like ions of abundant elements from $\mathrm{Cv}$ to $\mathrm{SxV}$ in the X-ray spectra of active regions observed by groups at the Naval Research Laboratory, Aerospace Corporation, Culham Laboratory and the University of Leicester. The same transitions have been observed in the spectra of flares (Neupert and Swartz, 1970) for ions up to Fexxv. Gabriel and Jordan show that for sufficiently high densities, the relative populations of $2{ }^{3} S$ and $2{ }^{3} P$ are strongly dependent on the electron density and therefore the intensity ratio $I\left(2{ }^{3} S-1{ }^{1} S\right) / I\left(2{ }^{3} P_{1}-1{ }^{1} S\right)$ can be used to determine the electron density, provided the rates for all relevant processes are known. If it can be assumed that each ion is radiating at the temperature at which its fractional concentration is highest, the electron density may be related to the temperature. It has been found in this way that solar active regions are inhomogeneous in both temperature and density and that the two are strongly correlated in the sense that high density and high temperature go together. The most recent results (Freeman et al, 1970) of the analysis of data compiled from many sources, in which new values of the transition probabilities of the forbidden line have been used, are as follows:

$\begin{array}{cc}T(\mathrm{~K}) & N_{e}\left(\mathrm{~cm}^{-3}\right) \\ 2 \times 10^{6} & 7 \times 10^{10} \\ 3 \times 10^{6} & 6 \times 10^{11} \\ 4 \times 10^{6} & 1.5 \times 10^{12} \\ 5 \times 10^{6} & 3 \times 10^{12} \\ 6 \times 10^{6} & 5 \times 10^{12}\end{array}$

The emission measures may also be derived from the absolute intensities of the X-ray lines and used in conjunction with the electron densities to estimate the volumes of the emitting regions. From a single rocket flight, Freeman et al. (1970) found that the emission measures derived for active region lines formed at about $5 \times 10^{6} \mathrm{~K}$ were smaller by a factor of 5 than for quiet coronal lines formed at $1.5 \times 10^{6} \mathrm{~K}$. The resulting estimate for the volume of the high temperature region is about $5 \times 10^{23} \mathrm{~cm}^{3}$, which is that contained in a filament $30000 \mathrm{~km}$ long and $150 \mathrm{~km}$ in diameter. A 
magnetic field of about $400 \mathrm{G}$ would be required to contain such a hot, dense plasma. Similar results have been found in a more detailed study of the X-ray spectra of 3 active regions by Batstone et al. (1970) in which absolute line fluxes are used to construct a model of an active region consisting of 4 components with temperatures equal to $2.5,4.5,6.5$, and $8.5 \times 10^{6} \mathrm{~K}$, respectively.

If we now compare active region densities derived from the X-ray spectra with those determined by Withbroe from the absolute intensities of ultraviolet emission lines observed above the limb, we find that for the same value of the temperature, $T_{e}=2 \times 10^{6} \mathrm{~K}$, the X-ray data give densities about 2 orders of magnitude greater than the ultraviolet data. We note however that the $\mathrm{X}$-radiation has been observed against the disk and therefore comes from the densest parts of the active regions whereas the ultraviolet emission is measured at an average height of 2 arc min above the limb.

In conclusion, I want to comment briefly on the special need for laboratory and theoretical data of all kinds to assist in the interpretation of the rapidly accumulating volume of observations in the X-ray region of the solar spectrum. The analysis of these data will be vital to the understanding of the physics of solar active regions and flares and is a necessary prelude to the interpretation of the spectra of galactic and extragalactic $X$-ray sources, which are soon to be observed. The spectral region from 1.2-20 $\AA$ has already been shown to include the hydrogen-like and helium-like spectra of $\mathrm{Ca}, \mathrm{S}, \mathrm{Si}, \mathrm{Al}$, and $\mathrm{Mg}$. Other lines such as those of FexviII-XXIV have been identified, not with the aid of laboratory wavelengths, but from calculated values based on screening corrections to hydrogenic levels. A large amount of basic data is needed before diagnostic techniques can be applied to the interpretation of the spectra.

Of special interest in this part of the spectrum is a strong feature at $1.9 \AA$ which is observed in the spectra of solar flares. From measurements obtained with OSO-III, Neupert identified the peak with the $1.87 \AA$ line of Fexxv $1 s^{2}-1 s 2 p$. New measurements of the $1.9 \AA$ feature by Neupert and Swartz (1970) from OSO-V show that it

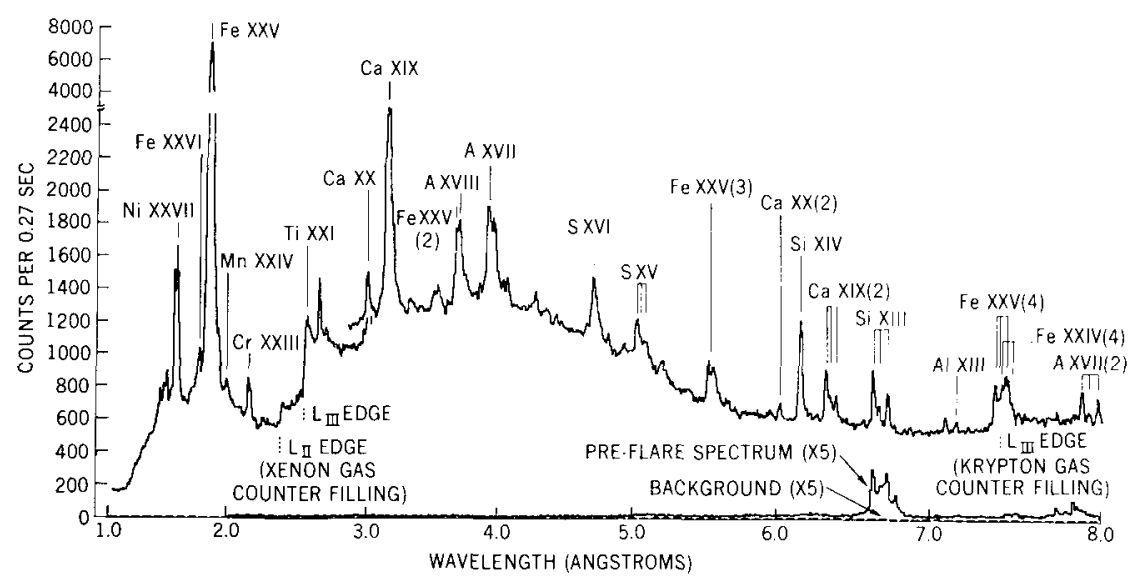

Fig. 4. X-ray spectrum of the solar flare of 1969 Feb. 27 recorded on-board the OSO-V satellite. [Neupert and Swartz, 1970.] 
is considerably more complex. Figure 4, reproduced from their paper, shows the spectrum of the flare of February 27, 1969. A number of triplet structures are present, representing the resonance, intersystem and forbidden lines of helium-like ions SixIII, Sxv, ArXVII, and CaxIX, the last two of which are also seen in the second order. The feature at $1.9 \AA$ is also seen with improved resolution in the second, third, and fourth orders and as a result is found to have the same triplet structure plus some additional components tentatively identified as $1 s^{2} 2 s-1 s 2 s 2 p$ transitions in FexXIV and $1 s^{2} 2 s^{2}-$ $1 s 2 s^{2} 2 p$ in FexxIII. The lithium-like Fe XxIv lines are surprisingly strong and probably represent the end product of dielectronic recombination from Fexxv. The intensity of the lithium-like transition $1 s^{2} 2 s-1 s 2 s 2 p$ relative to the helium-like transition $1 s^{2}-1 s 2 p$ of the same element is expected to increase in intensity with increasing nuclear charge if the upper level is populated by dielectronic recombination (Walker and Rugge, 1970).

\section{Acknowledgement}

We wish to acknowledge the support of the National Aeronautics and Space Administration, Contract Nas 5-9274 and Grant NGL-22-007-006.

\section{References}

Athay, R. G.: 1966, Astrophys. J. 145, 784.

Batstone, R. M., Evans, K., Parkinson, J. H., and Pounds, K. A.: 1970, Solar Phys. $13,389$.

Burton, W. M., Jordan, C., Ridgeley, A., and Wilson, R.: 1971, Phil. Trans. Roy. Soc. A. $270,81$. Cuny, Y.: 1970, Preprint.

Freeman, F. F., Gabriel, A. H., Jones, B. B., and Jordan, C.: 1971, Phil. Trans. Roy. Soc. A. $270,127$. Gabriel, A. H. and Jordan, C.: 1969, Nature 221, 947.

Gingerich, O.: 1970, IAU Symposium 36.

Gingerich, O., Cuny, Y., Kalkofen, W., and Noyes, R. W.: 1971, Solar Phys. 18, 347.

Henze, W., Jr.: 1969, Solar Phys. 9, 65.

Linsky, J. L. and Avrett, E. H.: 1970, Publ. Astron. Soc. Pacific 82, 169.

Neupert, W. M. and Swartz, M.: 1970, Astrophys. J. 160, L189.

Noyes, R. W.: 1971, in Macris (ed.), Physics of the Solar Corona (in press).

Noyes, R. W. and Kalkofen, W.: 1970, Solar Phys. 15, 120.

Noyes, R. W., Withbroe, G. L., and Kirshner, R. P.: 1970, Solar Phys. 11, 388.

Walker, A. B. C. and Rugge, H. R.; 1970, preprint.

Withbroe, G. L.; 1970a, Solar Phys. 11, 42.

Withbroe, G. L.: 1970b, Solar Phys. 11, 208.

Withbroe, G. L.: 1971, Solar Phys. 18, 458. 